Acta Crystallographica Section D

Biological Crystallography

ISSN 0907-4449

M. Bahar, ${ }^{a}$ C. Ballard,

S. X. Cohen, ${ }^{c}$ K. D. Cowtan, ${ }^{d}$

E. J. Dodson, ${ }^{d}$ P. Emsley, ${ }^{d}$

R. M. Esnouf, ${ }^{a}$ R. Keegan, ${ }^{b}$

V. Lamzin, ${ }^{e}$ G. Langer,

V. Levdikov, ' F. Long, d

C. Meier, ${ }^{a}$ A. Muller, ${ }^{d}$

G. N. Murshudov, ${ }^{\text {d }}$ A. Perrakis, ${ }^{c}$

C. Siebold, ${ }^{a}$ N. Stein, ${ }^{b}$

M. G. W. Turkenburg, ${ }^{d}$

A. A. Vagin, ${ }^{d}$ M. Winn,

G. Winter ${ }^{b}$ and K. S. Wilson ${ }^{d}$ *

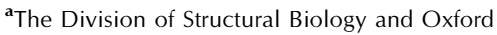
Protein Production Facility, The Wellcome Trust Centre for Human Genetics, Roosevelt Drive, Oxford OX3 7BN, England, ${ }^{\mathbf{b}} \mathrm{CCLRC}$, Daresbury Laboratory, Warrington WA4 4AD, England,

${ }^{\mathrm{c}}$ Netherlands Cancer Institute, Molecular

Carcinogenesis Department, Plesmanlaan 121, 1066 CX Amsterdam, The Netherlands, 'York Structural Biology Laboratory, Department of Chemistry, University of York, Heslington, York YO10 5YW, England, and ${ }^{\mathrm{E}} \mathrm{EMBL}$

Hamburg, Building 25A, DESY, Notkestrasse 85, 22603 Hamburg, Germany

Correspondence e-mail: keith@ysbl.york.ac.uk

\title{
SPINE workshop on automated X-ray analysis: a progress report
}

The Structural Proteomics In Europe (SPINE) consortium contained a workpackage to address the automated X-ray analysis of macromolecules. The aim of this workpackage was to increase the throughput of three-dimensional structures while maintaining the high quality of conventional analyses. SPINE was able to bring together developers of software with users from the partner laboratories. Here, the results of a workshop organized by the consortium to evaluate software developed in the member laboratories against a set of bacterial targets are described. The major emphasis was on molecular-replacement suites, where automation was most advanced. Data processing and analysis, use of experimental phases and model construction were also addressed, albeit at a lower level.

\section{Introduction}

The Structural Proteomics In Europe (SPINE) project, initiated in 2002, aimed to introduce new technologies and approaches to the complete set of processes required to determine the three-dimensional structures of biomedically relevant proteins. It was envisaged that the majority of these structures would be determined using X-ray crystallography and a distinct section of the programme was devoted to this method. The stated aims of this work package were

to address the problems of automated X-ray analysis of macromolecules. To achieve throughput in keeping with genome sequencing projects, macromolecular crystallography (MX) procedures must be streamlined, and work in a number of laboratories in Europe, including several SPINE partners, is directly addressing this. Scripting will link the various stages, and better algorithms will be formulated in key areas such as molecular replacement (MR), experimental phasing, automated generation of atomic models, molecular graphics and quality assessment. The software will ensure that high quality will accompany high throughput.

(from the SPINE Contract QLG2-CT-2002-00988).

Within the SPINE project, most of the resources were devoted to major bottlenecks for structural biology, namely in protein cloning, overexpression and crystallization. Hence, SPINE had only limited resources to contribute to the development of high-throughput crystallographic computing, but by bringing together major users and providers of code it was in a good position to gain access and provide some input to developments. This problem is being addressed worldwide. It is clear that contacts and coordination are essential to optimize the output of developers and that such contacts must be maintained. Early in the programme SPINE held two workshops to discuss automation, attended by people both within the project and from associated groups. This report
Received 22 February 2006 Accepted 14 August 2006
(C) 2006 International Union of Crystallography Printed in Denmark - all rights reserved 
Table 1

Targets used during the workshop.

$N_{\text {Res }}$ is the number of residues per molecule. $N_{\text {Mol }}$ is the expected number of molecules in the asymmetric unit. MR, molecular replacement. SAD, singlewavelength anomalous dispersion. MAD, multiple-wavelength anomalous dispersion.

\begin{tabular}{|c|c|c|c|c|c|c|}
\hline ID & $N_{\text {Res }}$ & $N_{\text {Mol }}$ & $\begin{array}{l}\text { Space } \\
\text { group }\end{array}$ & $\begin{array}{l}\text { Resolution } \\
(\AA)\end{array}$ & Method & $\begin{array}{l}\text { PDB } \\
\text { code }\end{array}$ \\
\hline \multicolumn{7}{|l|}{ York } \\
\hline BA0288 & 161 & 8 & $C 2$ & 1.80 & MR & $1 \mathrm{xmp}$ \\
\hline BA0296 & 346 & 2 & $P 4_{1}$ & 2.31 & MR & \\
\hline BA0592 & 377 & 6 & $C 2$ & 2.84 & MR & \\
\hline BA1071 & 311 & 1 & $P 2_{1} 2_{1} 2_{1}$ & 2.60 & MR & \\
\hline BA1483 & 235 & 8 & $P 2_{1} 2_{1} 2_{1}$ & 2.24 & MR & 1 xe3 \\
\hline BA 1563 & 282 & 2 & $P 2_{1}$ & 2.20 & MR & \\
\hline BA3935_1 & 292 & 4 & $P 2_{1} 2_{1} 2_{1}$ & 1.94 & MR & \\
\hline BA3935_2 & 292 & 4 & $P 2_{1} 2_{1} 2_{1}$ & 2.23 & MR & \\
\hline BA4499 & 203 & 2 & $P 2_{1}$ & 1.80 & MR & \\
\hline BA4508 & 298 & 1 & $C 2$ & 2.57 & MR & \\
\hline BA5696 & 208 & 2 & $P 2_{1}$ & 1.80 & MR & \\
\hline BA5705 & 327 & 2 & $P 4$ & 1.80 & MR & \\
\hline BSAppA & 543 & 1 & $P 2_{1} 2_{1} 2_{1}$ & 2.28 & MR & $1 \mathrm{xoc}$ \\
\hline BSYloQ & 298 & 1 & $P 4_{3} 2_{1} 2$ & 2.51 & MR & $1 \mathrm{t} 9 \mathrm{~h}$ \\
\hline CJ0982 & 292 & 2 & $C 2$ & 2.00 & MR & \\
\hline Peb3 & 230 & 2 & $P 2_{1} 2_{1} 2_{1}$ & 1.65 & MR & \\
\hline SiaP & 306 & 2 & $P 2_{1} 2_{1} 2$ & 2.60 & MAD & \\
\hline \multicolumn{7}{|l|}{ Oxford } \\
\hline OPPF651 & 294 & 2 & $P 2$ & 2.40 & MR & \\
\hline OPPF1294 & 193 & 2 & $P 4_{1} 2_{1} 2$ & 2.70 & MAD & \\
\hline OPPF1311 & 255 & 4 & $P 6_{1} 22$ & 2.72 & SAD & \\
\hline OPPF1314 & 200 & 2 & $P 1$ & 1.50 & MR & \\
\hline OPPF2088 & 139 & 1 & $P 3_{1} 21$ & 2.20 & MAD & \\
\hline OPPF 2153 & 222 & 4 & $P 2_{1}$ & 2.70 & MAD & \\
\hline OPPF2245 & 229 & 2 & $P 2_{1}$ & 3.30 & MR & \\
\hline
\end{tabular}

summarizes the activities at a third workshop where the current methodology was tested against targets selected from SPINE partner laboratories in Oxford and York. It does not describe in detail the software being developed or the structures of the individual targets, as these will be published elsewhere.

The SPINE project has tried to follow the traditional CCP4 (Collaborative Computational Project, Number 4, 1994) approach of linking contributions from a number of sources [such as $A R P / w A R P$ (Perrakis et al., 1999) and SHELX (Schneider \& Sheldrick, 2002)] to form a set of modular tools. This requires agreement on exchange protocols, which can be hard to establish, but will result in more robust and flexible software which can be easily upgraded in the years to come. The aim of the workshop was to assess progress towards this within the SPINE team and its associates.

\section{The target data sets}

23 data sets were selected from a group of bacterial (mainly Bacillus anthracis and Campylobacter jejuni; Alzari et al., 2006) targets under study in Oxford and York (Table 1). Merged structure factors and the amino-acid sequence data were the basis for most of the activity; however, for a subset of targets raw images were made available for assessment of an automated processing protocol. In the event, this effort was essentially restricted to a single problem data set (OPPF1314).
The basic selection parameters were that target proteins should be less than $50 \mathrm{kDa}$, not part of a complex, contain no signal peptides and have no transmembrane regions. Most were candidates for $\mathrm{MR}$ and were straightforward targets for the subsequent application of the $A R P / w A R P$ packages of automated electron-density interpretation.

During the workshop two structures were examined in greater detail to pinpoint problems in the structureautomation pipelines. These were OPPF1314 (Oxford) and SiaP (York).

\subsection{OPPF1314}

OPPF1314 data were used both for testing the data processing and analysis pipeline and for the automated modelbuilding procedures. OPPF1314 is a 5-formyltetrahydrofolate cycloligase (BA4489) and has a molecular weight of $22.3 \mathrm{kDa}$ (292 residues). The protein catalyses the ATP-dependent formation of 5,10-methenyltetrahydrofolate from 5-formyltetrahydrofolate (folinic acid; Huennekens et al., 1984).

The full details of the structure determination will be described elsewhere (Meier et al., 2006). Briefly, crystals were obtained from cocrystallizations of OPPF1314 with the substrates ATP and 5-formyltetrahydrofolate and data extending to $1.5 \AA$ resolution were measured on ID14EH1 at the ESRF from a crystal belonging to space group $P 1$ containing two molecules in the crystallographic asymmetric unit. Data were acquired in a high-resolution pass (in which many of the low-resolution reflections were overloaded) followed by a low-resolution pass. The diffraction showed a high degree of mosaicity. Data reduction with DENZO/ $S C A L E P A C K$ (Otwinowski \& Minor, 1997) prior to the workshop gave an apparently reasonable merged data set, but it had proved impossible to phase this satisfactorily by MR using either a medium-resolution model structure obtained previously in a different space group or a related structure (PDB code $1 \mathrm{ydm}$ ) with $47 \%$ sequence identity.

\subsection{SiaP}

The SiaP protein, a candidate for MAD phasing (Table 1), was used during the workshop to test using experimental phasing to kick-start automated model building. One structure in the PDB, $1 \mathrm{k} 7 \mathrm{k}$, had some (25\%) sequence identity, but only over a third of the molecule. MAD data sets had been collected for SeMet-labelled protein at three wavelengths $(0.97907,0.90778$ and $0.97920 \AA$ ) on BM14, the UK MAD beamline at the ESRF. The SeMet crystals diffracted to $2.6 \AA$ resolution with high merging $R$ factors in the outer ranges and belonged to space group $P 2_{1} 2_{1} 2$, with two molecules in the crystallographic asymmetric unit. Although the data between 2.9 and $2.6 \AA$ resolution were especially weak $[I / \sigma(I)=1.5$ in the outer shell], they proved essential for structure solution.

$16 \mathrm{Se}$ atoms were expected in the asymmetric unit and SHELXC and SHELXD (Schneider \& Sheldrick, 2002) found 14 sites. Phases had been calculated with SHELXE but automated construction of a model using REFMAC-ARP/ $w A R P$ from these phases had failed: the procedure built many 
short disconnected peptides with no side chains docked. The same heavy-atom solution was used with greater success in RESOLVE (Terwilliger, 2003), which built 468 residues in $\sim 44$ chains, but with only 75 side chains docked. This model was in turn fed to REFMAC-ARP/wARP with the 'default' options, but the procedure dismembered the model rather than adding additional features. The situation was improved by using the RESOLVE model with phase restraints imposed during $R E F M A C$ refinement cycles. For this purpose, the reference phase set was based on the original SHELXE phases from the selenium substructure, improved by solvent flattening to give a single 'best' phase estimate with an associated figure of merit. This gave a better result, with $R$ converging at around $30.4 \%$ ( $R_{\text {free }}$ was not used) for a model with 260 backbone residues in 27 chains and 65 side chains docked. This again reflected some degradation of the RESOLVE model. All this work was carried out prior to the workshop.

\section{Software suites and developers involved}

Extensive use was made of $C C P 4$ modules and utilities. The $X I A-D P A$ system was used for the processing of $\mathrm{X}$-ray images and their subsequent analysis and quality assessment. $X I A$ $D P A$ provides wrappers for software packages including LABELIT (Sauter et al., 2004), MOSFLM (Leslie, 1999), XDS and XSCALE (Kabsch, 1993), SCALA (Evans, 1993), TRUNCATE (French \& Wilson, 1978) and SFCHECK (Vaguine et al., 1999).

Sequence analysis and putative MR model identification used well established software packages available on the web, particularly MSDtarget and MSDfold from EBI (http:// www.ebi.ac.uk/msd) and BLAST (http://www.ncbi.nlm.nih.gov/ BLAST/).

MR was carried out by three teams. All used established core software, but different scripted protocols. One group (RK and MW) has developed $M r B U M P$, which uses existing web servers (described below) to select multiple models, modifies them using CHAINSAW (Stein, unpublished work), MOLREP (Vagin \& Teplyakov, 1997) or PDBCLIP (a local utility), followed by application of MOLREP or Phaser (McCoy et al., 2005) for the MR search. Models were assessed using REFMAC (Murshudov et al., 1997). A second group (NS and $\mathrm{CB}$ ) has developed AutoAMoRe, incorporating CHAINSAW and AMoRe (Navaza, 1994). The third group (GNM, FL and AAV) has developed a package BALBES that utilizes a pre-constructed database for model selection, SFCHECK for data-quality analysis, MOLREP for model preparation and molecular replacement, and $R E F M A C$ for initial refinement and final quality assessment. Sequence analysis and model identification use programs and procedures under development by the authors (Murshudov, private communication).

With the exception of SiaP, there was little work on experimental phasing, as the automated pipelines are at an earlier stage of development. However, several of the Oxford structures requiring experimental phasing had been solved prior to the workshop using SHELXD and SHELXE (Schneider \& Sheldrick, 2002).

Model construction and rebuilding for both $\mathrm{MR}$ and experimentally phased maps was primarily based on the REFMAC-ARP/wARP pipeline (SC, GL; Perrakis et al., 1999). Maps were visualized using Coot (Emsley \& Cowtan, 2004) and development versions of Pirate (Cowtan, 2000) and Buccaneer (Cowtan, 2001) were tested. Ligand fitting was attempted using both $A R P / w A R P$ and Coot.

\section{Data processing}

\subsection{Data integration}

$X I A-D P A$ was applied to targets where images were available. $X I A-D P A$ is an automated wrapper for existing data-processing and analysis software. It aims to combine independently developed functionality in a modular manner so that it will be straightforward to replace individual functions. $X I A-D P A$ incorporates these into an expert system capable of making decisions about how to process data without user intervention.

The user interface to XIA-DPA is simple: the filename of an image is sufficient to initiate data processing tasks for either two-dimensional or three-dimensional integration: xiaautoprocess-2d /path/to/data/set/foo_1_001.img or xia-autoprocess-3d /path/to/data/set/foo_1_001.img.

The current software distribution uses LABELIT to perform the autoindexing followed by two-dimensional integration with MOSFLM or three-dimensional integration using XDS. POINTLESS (Evans, 2006) is used to select the most likely point group. Scaling and merging can be performed with $S C A L A$ and TRUNCATE or by XSCALE. Images are processed to provide reduced and scaled, merged and unmerged reflection files in the commonly useful formats (MTZ with $I^{+}, I^{-}, I, F^{+}, F^{-}, F$ and $S C A L E P A C K$ ) along with estimates of the resolution and lists of possible space groups from an analysis of the systematic absences. The objective is to provide the initial stages of a 'data-to-structure' pipeline to generate machine-readable information to be used in the subsequent steps of structure solution.

\subsection{Data analysis and quality assessment}

It was realised very early in the workshop that the experimental data as provided often did not carry all of the necessary crystal information in a form accessible by user or computer. Some of the information such as wavelength should be recorded in the reflection-file header. We suggest that a simple solution would be to define an accepted exchange format and record tagged information conforming to this format in an exchange file.

Decisions to be taken in automatic procedures fall into four categories.

(i) Sample parameters, e.g. the sequence, molecular weight and expected numbers of 'heavy' atoms.

(ii) Details of the X-ray experiment: direct parameters such as wavelength, beamline and temperature, derived parameters 
including unit cell, point group, the likely number of molecules in the crystallographic asymmetric unit and the presence of any non-crystallographic translational operator and quality indicators including nominal resolution, estimated $B$ factor and anisotropy plus completeness at both low and high reso-

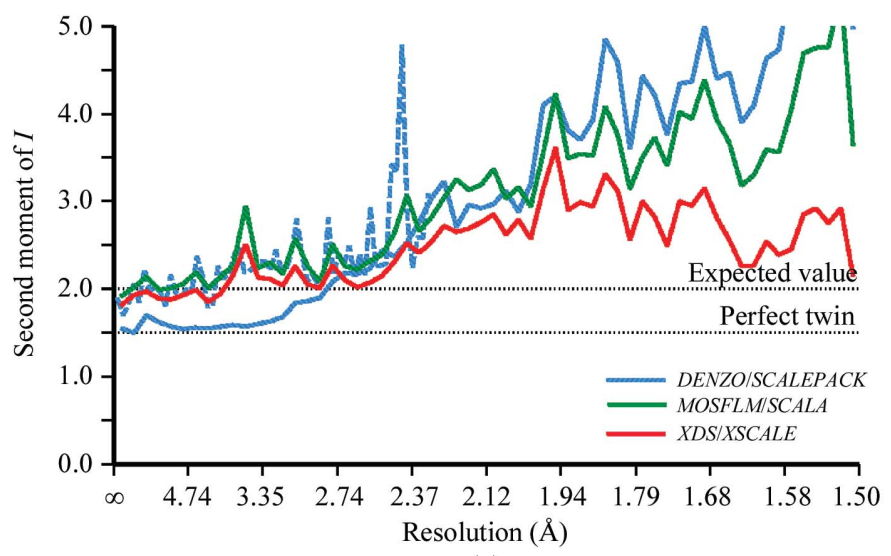

(a)

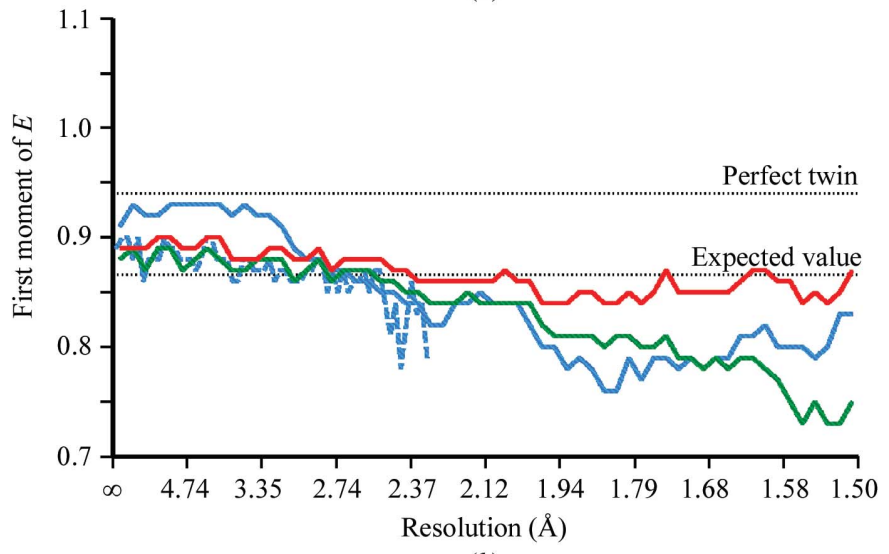

(b)

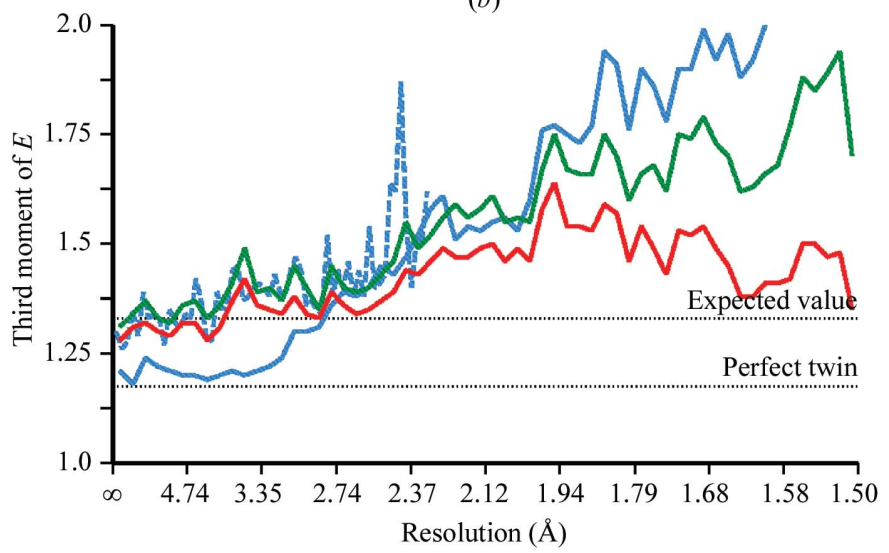

(c)

Figure 1

Results of data reduction for OPPF1314 images using different dataprocessing packages (see Table 3) analysed with TRUNCATE. (a) Second moments of $I$ in resolution shells. (b) First moments of $E$ in resolution shells. (c) Third moments of $E$ in resolution shells. In all panels the combined data sets are shown with solid lines (blue, DENZO/ $S C A L E P A C K$; green, MOSFLM/SCALA; red, XDS/XSCALE), while results for the low-resolution pass processed with DENZO/SCALE$P A C K$ are shown by blue dashed lines. lution (the former being important for MR), multiplicity, $I / \sigma(I)$ and merging $R$ factor, all as functions of resolution.

(iii) Intensity statistics to be tested against expectation values including cumulative intensity distributions and moments. These are sensitive indicators of problems in the experiment such as twinning or local errors in the processing such as saturation of substantial numbers of low-resolution terms (Fig. 1).

(iv) The identification of special features of the crystal such as pseudo-symmetry or potential alternative indexing.

This list is certainly not complete and requires agreement amongst the community on a formal definition of needs.

Most of the necessary information is already available in the output from various programs, but is not yet encoded into an accepted exchange file. The data sets used for the workshop were evaluated retrospectively using TRUNCATE and SFCHECK (Table 2).

\subsection{Test applications}

4.3.1. OPPF1314. Analysis of the merged OPPF1314 data had shown an unusual distribution of reflection intensities, in particular in the low-resolution range (Fig. 1). The original images were reprocessed during the workshop with XIA$D P A$, trying both the two-dimensional and three-dimensional options. The two-dimensional pipeline (i.e. using MOSFLM and $S C A L A$ ) produced similar results to those from DENZO/ $S C A L E P A C K$; again the intensity statistics were unusual. However, the three-dimensional pipeline ( $X D S$ and $X S C A L E)$ gave a well merged data set which led to a successful structure solution in a straightforward manner. More detailed analysis and careful reprocessing with MOSFLM/SCALA subsequent to the workshop suggested that the first failures were a consequence of poor relative scaling of the low- and high-resolution passes and that the high mosaic spread proved difficult to handle with the current twodimensional software. The scaling $R$ factor between the $X D S$ and reprocessed MOSFLM amplitudes is $\sim 4 \%$ to $2.3 \AA$, rising to $16 \%$ at $1.5 \AA$. The quality assessment (Tables 2 and 3 ) suggested that the two-dimensional integration with MOSFLM was unsatisfactory in the higher resolution ranges. SFCHECK showed that the high mosaicity reduced the completeness of the two-dimensional data set in certain zones.

4.3.2. Other targets. Diffraction images for a further six targets were reprocessed at the workshop using XIA-DPA using the two-dimensional option (Table 4). The agreement with the data provided for the workshop for BA0592 appears to be satisfactory and the quality assessment for the other data sets was acceptable (Table 2). BA0296 illustrates a situation where rapid automated data processing should have been performed during data collection to select the best strategy. Autoindexing was satisfied by a cubic crystal class. However, subsequent analysis showed that the point group was tetragonal. This unfortunately resulted in a rather incomplete data set. This workshop greatly stimulated the further development of the XIA-DPA pipeline. 


\subsection{Discussion; lessons for automated data processing}

The data-processing and analysis step is critical for the structure-solution pipeline. The XIA-DPA pipeline performs adequately for many data sets, but it is essential that it flags aberrant cases and alerts the crystallographer in the more challenging cases, such as OPPF1314. In the case of BA0296 the information derived from initial indexing was later found to be incorrect and this highlights the need for easy and reliable determination of the point group from limited data during data collection.

A final assessment of data quality at the end of an automated procedure is good practice and should always be carried out. Automated procedures have two features to offer, reproducibility and standardization, which should allow for more objective summary statistics. They may also perform the tedious transformations between different packages, enabling all statistics to be calculated by the same program and hence be more comparable.

Finally, in any automation effort, the success of the pipeline depends on the cumulative success of the individual steps. As data-processing programs become more reliable and sophisticated, the overall success rate of the pipeline should improve.

\section{Molecular-replacement pipelines}

Of all the automated structure-solution pipelines, those addressing MR are the most advanced at present and were the core activity at the workshop. Each pipeline has five basic tasks to address.

(i) Is there a suitable model structure in the PDB? This requires the use of sequence-matching tools, such as $B L A S T$ and FASTA (Brenner et al., 1998), which can scan the PDB (Bernstein et al., 1977; Berman et al., 2000) for homologous structures to use as templates. The results of these alignments have to be viewed critically. For example, sequence similarity over a short segment is likely to be of limited value; what is required is a fit over the whole length of the sequence or at least over an extensive fraction such as a domain. Decisions must be made about the optimum search unit based on knowledge of the biology: this ranges from identifying possible oligomers for the search to breaking down a single chain into individual domains. Models can often be usefully modified

Table 2
$N(I)$ is the cumulative intensity distribution from TRUNCATE, flagged as ' $\mathrm{OK}$ ', 'sig' (sigmoidal, indicating twinning) or 'odd'. $B_{\text {est }}$ are the overall $B$-factor estimates by each program (TRUNCATE and SFCHECK, (he degree of anisotropy in the merged data: this is described in SFCHECK the three eigenvalues of the overall anisotropic scaling ellipsoid. An off-origin peak in the native lags this. The likelihood of twinning is estimated by $S F C H E C K$, after taking account of pseudo-translation and anisotropy. Substantial deviation from linearity of the Wilson plot is flagged by TRUNCATE.

\begin{tabular}{|c|c|c|c|c|c|c|c|}
\hline ID & $N(I)$ & $\begin{array}{l}B_{\text {est }} \\
\left(\AA^{2}\right)\end{array}$ & Aniso & PseudoT & Twin & Wilson & Comments \\
\hline \multicolumn{8}{|l|}{ York } \\
\hline BA0288 & OK & $18 / 27$ & $\mathrm{~N}$ & & & & Low-res. weak \\
\hline BA0296 & Sig & $58 / 66$ & $\mathrm{~N}$ & & $\mathrm{~T}$ & & High-res. incomplete \\
\hline BA0592 & Odd & $71 / 69$ & $\mathrm{Y}$ & & & & $95 \%$ complete \\
\hline BA1071 & OK & $54 / 55$ & $\mathrm{Y}$ & & & & $95 \%$ complete, low-res. weak \\
\hline BA1483 & OK & $27 / 35$ & $\mathrm{Y} ?$ & & & & $\begin{array}{l}\text { Low-res. weak, } \\
\text { high-res. incomplete }\end{array}$ \\
\hline BA1563 & OK & $35 / 44$ & $\mathrm{Y}$ & $\mathrm{Y}$ & & & $\begin{array}{l}\text { Low-res. weak, } \\
\text { high-res. incomplete }\end{array}$ \\
\hline BA3935_1 & OK & $20 / 29$ & $\mathrm{~N}$ & & & Nonlinear & Low-res. weak \\
\hline BA3935_2 & OK & $42 / 49$ & $\mathrm{Y}$ & & & & $\begin{array}{l}\text { Low-res. weak, } \\
\text { better than previous }\end{array}$ \\
\hline BA4499 & Sig & $25 / 34$ & $\mathrm{Y}$ & & & & \\
\hline BA4508 & Odd & $53 / 56$ & $\mathrm{Y}$ & & & & $94 \%$ complete, poor strategy? \\
\hline BA5696 & $\mathrm{OK}$ & $27 / 36$ & $\mathrm{Y}$ & & & & \\
\hline BA5705 & Sig & $24 / 32$ & $\mathrm{Y}$ & & $\mathrm{T}$ ? & & \\
\hline BSAppA & OK & $32 / 39$ & $\mathrm{Y}$ & & & & Low-res. weak \\
\hline BSYloQ & $\mathrm{OK}$ & $44 / 66$ & $\mathrm{Y}$ & & & & \\
\hline CJ0982 & Sig & 2738 & $\mathrm{Y}$ & & & Nonlinear & $\begin{array}{l}\text { Incomplete and very } \\
\text { anisotropic }\end{array}$ \\
\hline Peb3 & OK & $17 / 24$ & $\mathrm{Y}$ & & & Odd & Low-res. odd, probably OK \\
\hline SiaP & $\mathrm{OK}$ & $34 / 42$ & $\mathrm{Y}$ & & & & Low-res. weak \\
\hline \multicolumn{8}{|l|}{ Oxford } \\
\hline OPPF651 & Wild & $41 / 47$ & $\mathrm{Y}$ & $\mathrm{Y}$ & & & Low-res. weak \\
\hline OPPF1294 & Sig & $105 / 105$ & $\mathrm{~N}$ & & $\mathrm{~T}$ ? & & Very weak overall \\
\hline OPPF1311 & Odd & $72 / 70$ & $\mathrm{Y}$ & $\mathrm{Y}$ & & & An odd distribution \\
\hline OPPF1314 & OK & $37 / 47$ & $\mathrm{Y}$ & & & & $\begin{array}{l}\text { See text; incomplete, } \\
\text { problems in merging }\end{array}$ \\
\hline OPPF2088 & Sig & $42 / 51$ & $\mathrm{Y}$ & & $\mathrm{T}$ ? & & \\
\hline OPPF2153 & Wild & $49 / 50$ & $\mathrm{Y}$ & $\mathrm{Y}$ & $?$ & Nonlinear & Data very weak \\
\hline OPPF2245 & OK & $76 / 54$ & $\mathrm{Y}$ & & $\mathrm{T}$ ? & Nonlinear & Low resolution \\
\hline \multicolumn{8}{|c|}{$X I A-D P A$ processing } \\
\hline OPPF1314-2D & Odd & $24 / 19$ & $\mathrm{Y}$ & & & OK & $5 \%$ of reflections rejected \\
\hline OPPF1314-3D & OK & $22 / 19$ & $\mathrm{Y}$ & & & OK & $\begin{array}{l}\text { Fewer reflections rejected, } \\
\text { data quality better }\end{array}$ \\
\hline BA0296 & Sig & $49 / 53$ & $\mathrm{~N}$ & $\mathrm{~N}$ & $\mathrm{~T}$ & & $\begin{array}{l}\text { Missing wedge of } \\
\text { data, incomplete }\end{array}$ \\
\hline BA0592 & OK & $69 / 58$ & $\mathrm{Y}$ & $\mathrm{N}$ & & & Very anisotropic \\
\hline BA1071 & Sig & $34 / 44$ & $\mathrm{Y}$ & $\mathrm{N}$ & $\mathrm{T}$ ? & & Indications of twinning \\
\hline BA2236 & Sig & $26 / 39$ & $\mathrm{Y}$ & $\mathrm{N}$ & $\mathrm{T}$ ? & & Wilson poor for high res. \\
\hline BA4525 & OK & $21 / 33$ & $\mathrm{Y}$ & $\mathrm{N}$ & & & Data missing from $2.7 \AA$ \\
\hline BA5505 & OK & $57 / 66$ & $\mathrm{~N}$ & $\mathrm{~N}$ & & & Ice ring \\
\hline
\end{tabular}

based on the sequence alignment, e.g. the deletion of gaps and pruning of side chains. A practical advantage of such pruning is to introduce the 'correct' residue numbering and naming into the model, which is useful during rebuilding.

(ii) What is the information content of the X-ray data? The reduction and analysis of the X-ray images should both provide a set of essential information and make it available to the user/program/pipeline (see $\$ 4.2$ ).

(iii) Does the MR search indicate a satisfactory solution? Each program provides a scoring function for potential solutions and a contrast between the best and the others is required. In some cases, the crystal space group is ambiguous; the automated translational searches must then cover all 
Table 3

Data sets obtained by processing of the ESRF ID14EH1 images obtained from OPPF1314 cocrystals (space group $P 1$ ) using different data-processing packages.

Images for high- and low-resolution (LOW) passes were collected and these were also merged to form combined (CMB) data sets. Data were processed using DENZO/SCALEPACK (DEN), MOSFLM/ $S C A L A$ (MOS) and XDS/XSCALE (XDS). Values in parentheses are for the outer (highest resolution) data shells, i.e. $2.38-2.30 \AA$ for the low-resolution pass (note that the low completeness results from processing into the corners of a square detector) and $1.55-1.50 \AA$ for the combined low- and highresolution passes.

\begin{tabular}{lllllll}
\hline & DEN-LOW & MOS-LOW & XDS-LOW & DEN-CMB & MOS-CMB & XDS-CMB \\
\hline Resolution limit (A) & 2.30 & 2.30 & 2.30 & 1.50 & 1.50 & 1.50 \\
Unique reflections & 11585 & 10987 & 11455 & 59140 & 56913 & 60271 \\
Completeness (\%) & $63.5(11.4)$ & $62.1(13.8)$ & $64.2(14.4)$ & $93.0(90.2)$ & $90.5(89.1)$ & $95.2(93.7)$ \\
Multiplicity & $2.4(2.2)$ & $2.4(2.3)$ & $2.4(2.3)$ & $2.1(1.7)$ & $2.4(1.9)$ & $2.4(2.0)$ \\
$I / \sigma(I)$ & $20.6(5.5)$ & $18.3(4.0)$ & 17.2 & $20.9(1.1)$ & $8.7(1.3)$ & $11.1(2.1)$ \\
$R_{\text {merge }}^{\dagger}(\%)$ & $4.0(17.5)$ & $3.9(17.7)$ & $3.2(15.5)$ & $5.3(83.5)$ & $5.7(63.9)$ & $4.3(46.7)$ \\
\hline
\end{tabular}

$\dagger R_{\text {merge }}=\sum I-\langle I\rangle / \sum\langle I\rangle$.

Table 4

Summary of other data sets processed with $X I A-D P A$.

For BA0592 a previously processed data set had also been supplied to the workshop (Table 1 ) and $R_{\mathrm{fac}}$ gives the scaling $R$ factor between this data set and the $X I A-D P A$ processing. The suggested space group is that predicted from systematic absence analysis.

\begin{tabular}{llllllll}
\hline Protein & $\begin{array}{l}\text { Resolution } \\
(\AA)\end{array}$ & $\begin{array}{l}\text { Space } \\
\text { group }\end{array}$ & $R_{\text {merge }}$ & $\begin{array}{l}\text { Completeness } \\
(\%)\end{array}$ & Multiplicity & I/ $\sigma(I)$ & $R_{\text {fac }}$ \\
\hline BA0296 & 2.5 & $P 4_{1} / 4_{3}$ & 11.3 & $81.7(70.9)$ & 2.5 & $7.7(2.3)$ & \\
BA0592 & 3.0 & $C 2$ & 20.1 & $99.5(99.3)$ & 4.0 & $8.4(1.9)$ & 0.087 \\
BA1071 & 2.0 & $P 2_{1} 2_{1} 2_{1}$ & 14.0 & $96.7(81.5)$ & 4.1 & $6.6(1.7)$ & \\
BA2236 & 2.1 & $P 2_{1} 2_{1} 2_{1}$ & 15.1 & $88.1(56.8)$ & 6.0 & $7.6(1.8)$ & \\
BA4525 & 2.0 & $C 2$ & 7.8 & $69.1(17.4)$ & 3.5 & $13.0(3.0)$ & \\
BA5055 & 2.4 & $P 3_{n} 21$ & 14.8 & $96.0(84.6)$ & 7.3 & $10.2(1.0)$ & \\
\hline
\end{tabular}

possibilities and a clear result in just one of these is also an indicator of likely success.

(iv) Is the solution likely to be correct? Firstly, for a correct solution the model molecule must not clash too severely with symmetry-related copies in the unit cell. Secondly, preliminary automated refinement should reduce both $R$ and $R_{\text {free. More }}$ sophisticated tests can address such questions as to whether the solution makes good biological sense (e.g. residues in suitable electrostatic environments, sensible lattice contacts), but these are harder to automate.

(v) Can the resulting model be satisfactorily rebuilt? The best criterion for a suitable MR solution remains the quality of the resulting electron density. If new correct features are visible in the map and incorrect features of the model are not, then it can be considered a solution. This is discussed in $\$ 7$.

\subsection{Individual pipelines}

In this section, we report on the results obtained by the three teams. All teams used some of the same tools for the various tasks. CHAINSAW (next release of the CCP4 suite) is a new utility developed for manipulating models. It examines the sequence alignment between target and template provided in a standard format and modifies the template PDB file by pruning non-conserved side chains back to the $\gamma$ atom while leaving conserved residues unchanged. Atom and residue names and numbers are matched to those in the target. The result is what Schwarzenbacher et al. (2004) have termed a 'mixed model', since more atoms are preserved than in a polyalanine model, but parts of the model which are unlikely to be present in the crystal structure, and thus may degrade the signal, are pruned.

MOLREP also contains many modelpreparation tools. It aligns a given sequence with the model and prepares a truncated model. It can also carry out locked translation searches using a given non-crystallographic symmetry transformation. $A M o R e$ is a well established package which separates the rotation, translation and rigid-body refinement modules, allowing great flexibility in tailoring protocol to problem. Phaser has a sophisticated scoring scheme and also recalculates the orientation search for multiple molecules, taking account of the contribution from any model positioned previously. REFMAC5 refinement was used to assess the quality of the solutions; if both the $R$ and $R_{\text {free }}$ fell, then a solution was judged to be substantially correct.

5.1.1. The MrBUMP package. The $M r B U M P$ package has been developed as part of the eHTPX (http://www. e-htpx.ac.uk/) and CCP4 projects: eHTPX provided extensive computing resources in the form of an 18-CPU cluster accessed via an eHTPX web service. This mode is particularly valuable for marginal cases with low sequence homology where a parallel approach can be used with a range of putative trial models and methodologies being investigated contemporaneously. Alternatively, the $M r B U M P$ package can be customized to run on a desktop and it was tested in both modes during the workshop. It is designed to make use of webaccessible databases of sequences and structures, rather than relying on local databases. This guarantees that the information is up to date, but has the drawback that queries are submitted across a public network and may be slower. Since the workshop, $M r B U M P$ has been extended to allow this search to be performed locally.

In brief, the $M r B U M P$ pipeline comprises the following steps: the properties of the target are generated from the reflection and sequence files provided (for example, the expected number of molecules in the asymmetric unit) and then a FASTA search of the current PDB is made to generate a list of possible homologous structures, which are then downloaded. For each, the PQS server (http://pqs.ebi.ac.uk/) is queried to ascertain whether the model exists as a multimer. If so, and if the multimer could fit into the target unit cell, it is added to the list of templates. A recent addition, not available for the tests described here, is to add domains identified by SCOP (Murzin et al., 1995) to the list of templates. 


\section{Table 5}

Summary of results from $M r B U M P$.

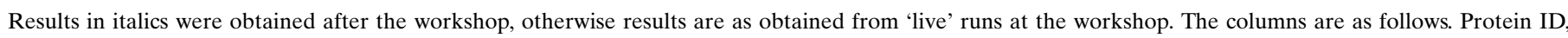

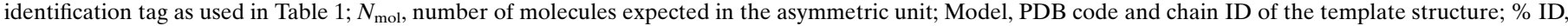

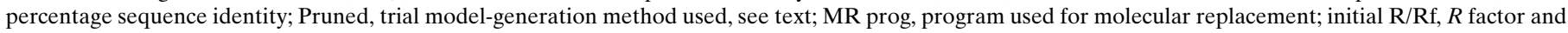

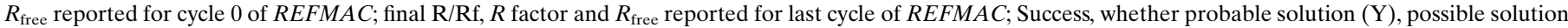
(P) or no solution (N); Rebuilt, whether rebuild attempted in $A R P / w A R P$ and if so whether successful.

\begin{tabular}{|c|c|c|c|c|c|c|c|c|c|}
\hline ID & $N_{\text {mol }}$ & Model & $\begin{array}{l}\% \\
\text { ID }\end{array}$ & Pruned & $\begin{array}{l}\text { MR } \\
\text { Prog }\end{array}$ & $\begin{array}{l}\text { Initial } \\
\mathrm{R} / \mathrm{Rf}\end{array}$ & $\begin{array}{l}\text { Final } \\
\text { R/Rf }\end{array}$ & Success & Rebuilt \\
\hline BA0288 & 8 & 1u11_A & 66 & MOLREP & MOLREP & $0.45 / 0.45$ & $0.30 / 0.33$ & $\mathrm{Y}$ & $\mathrm{Y}$ \\
\hline BA0288 & 8 & 1u11_0 & 66 & Multimer & $M O L R E P$ & $0.46 / 0.45$ & $0.32 / 0.36$ & $\mathrm{Y}$ & - \\
\hline BA0592 & 6 & 1pjb_A & 54 & PDBCLIP & $M O L R E P$ & $0.44 / 0.43$ & $0.32 / 0.39$ & $\mathrm{Y}$ & $\mathrm{N}$ \\
\hline BA1071 & 1 & $1 \mathrm{c} 9 \mathrm{e} \_A$ & 73 & CHAINSAW & $M O L R E P$ & $0.43 / 0.44$ & $0.29 / 0.36$ & $\mathrm{Y}$ & $\mathrm{Y}$ \\
\hline$B A 1483$ & 6 & 1pr1_A & 57 & CHAINSAW & $M O L R E P$ & $0.51 / 0.52$ & $0.35 / 0.41$ & $Y$ & $Y$ \\
\hline BA1563 & 2 & $1 \mathrm{ufv} \_B$ & 49 & CHAINSAW & $M O L R E P$ & $0.51 / 0.50$ & $0.40 / 0.47$ & $\mathrm{P}$ & $\mathrm{P}$ \\
\hline BA3935_1 & 4 & 1dhp_A & 42 & MOLREP & $M O L R E P$ & $0.52 / 0.52$ & $0.37 / 0.41$ & $\mathrm{Y}$ & $\mathrm{Y}$ \\
\hline BA3935_2 & 4 & $1 s 5 t \_B$ & 42 & MOLREP & MOLREP & $0.49 / 0.49$ & $0.33 / 0.39$ & $Y$ & $Y$ \\
\hline BA4499 & 2 & 1jr9_0 & 71 & Multimer & MOLREP & $0.44 / 0.46$ & $0.28 / 0.33$ & $\mathrm{Y}$ & $\mathrm{Y}$ \\
\hline BA4508 & 1 & 1qtw_A & 32 & CHAINSAW & MOLREP & $0.52 / 0.53$ & $0.40 / 0.48$ & $\mathrm{P}$ & $\mathrm{N}$ \\
\hline BA4508 & 1 & 1qtw_A & 32 & CHAINSAW & Phaser & $0.52 / 0.51$ & $0.40 / 0.48$ & $\mathrm{P}$ & - \\
\hline BA5696 & 2 & 1jr9_0 & 56 & Multimer & $M O L R E P$ & $0.46 / 0.47$ & $0.29 / 0.33$ & $\mathrm{Y}$ & $\mathrm{Y}$ \\
\hline BA5705 & 2 & $1 \mathrm{vrd} \_B$ & 35 & CHAINSAW & $M O L R E P$ & $0.55 / 0.56$ & $0.43 / 0.49$ & $\mathrm{P}$ & - \\
\hline BA5705 & 2 & 1vrd_B & 35 & CHAINSAW & Phaser & $0.54 / 0.55$ & $0.42 / 0.45$ & $\mathrm{Y}$ & $\mathrm{Y}$ \\
\hline BSAppa & 1 & 1dpe & 28 & CHAINSAW & MOLREP & $0.57 / 0.57$ & $0.56 / 0.55$ & $\mathrm{P}$ & - \\
\hline BSYloQ & 1 & 1u01_A & 40 & CHAINSAW & $M O L R E P$ & $0.55 / 0.52$ & $0.41 / 0.48$ & $\mathrm{P}$ & - \\
\hline BSYloQ & 1 & 1u0l_A & 40 & CHAINSAW & Phaser & $0.54 / 0.50$ & $0.42 / 0.49$ & $\mathrm{P}$ & - \\
\hline CJ0982 & 2 & 1qok_A & 44 & - & - & - & - & $\mathrm{N}$ & - \\
\hline OPPF651 & 2 & 1v6s_A & 51 & $M O L R E P$ & $M O L R E P$ & $0.66 / 0.65$ & $0.42 / 0.50$ & $\mathrm{Y}$ & $\mathrm{N}$ \\
\hline OPPF651 & 2 & $1 \mathrm{php}$ & 77 & MOLREP & MOLREP & $0.65 / 0.64$ & $0.33 / 0.39$ & $\mathrm{Y}$ & $\mathrm{Y}$ \\
\hline OPPF1314 & 2 & 1ydm_C & 47 & CHAINSAW & $M O L R E P$ & $0.53 / 0.52$ & $0.47 / 0.48$ & $\mathrm{P}$ & $\mathrm{P}$ \\
\hline OPPF2245 & 2 & 1lfp_A & 37 & CHAINSAW & $M O L R E P$ & $0.54 / 0.55$ & $0.45 / 0.52$ & $\mathrm{P}$ & $\mathrm{N}$ \\
\hline
\end{tabular}

The next step is to generate trial models from the templates. Currently, three methods are used. Firstly, in the PDBCLIP method the raw template coordinates are used, after various tidying steps such as removal of waters, removal of alternative conformations etc. Secondly, the alignment and modelimprovement method of $M O L R E P$ is used. Thirdly, the CCP4 program $C H A I N S A W$ described above is used to provide a mixed model.

The top models are passed to MOLREP for the first attempt at MR. If $M O L R E P$ produces a solution (irrespective of score), the positioned model is passed to REFMAC for 30 cycles of restrained refinement. The free $R$ factor is used as the criterion for success. If the $R_{\text {free }}$ drops significantly, the script stops and reports details of the solution. Marginal solutions are identified without stopping the script. Unless a clear solution is obtained, the $M r B U M P$ script continues to process all trial models through MOLREP, after which the process is repeated using Phaser as the MR engine. For the cluster implementation of $M r B U M P$, trial models are processed in parallel, with a success on any cluster node stopping the script on all nodes.

A summary of $M r B U M P$ results is given in Table 5. Unless explicitly indicated otherwise, these results were obtained during the workshop, without previous knowledge of the structures and without any ad hoc customization of the default script. The only exception to this is that if the actual target structure had been deposited, the script was run with this structure excluded. It became clear that the criterion for a good solution was too strict and in many cases the script continued processing trial models after a good solution had been found. In these cases, Table 5 shows one or more of the solutions identified as 'marginal' rather than as 'success'. For comparison, in some cases, the solution from the Phaser loop is shown alongside that from the MOLREP loop. The result of the MR step is a positioned but inaccurate and incomplete model. The cycles of restrained refinement often indicate that the model will refine and that a final model is likely to be realised (see, for example, BA4499). In other cases, there is no such clear-cut indication (for example, BA1563) and conclusions on the success of the procedure must await model rebuilding.

The second column in Table 5 shows the actual number of molecules in the asymmetric unit. In three cases, the automated script overestimated the correct number: BA1483, BA3935_2 and BA0592. For the last two this did not matter, since MOLREP failed to find the predicted last molecule and the refinement proceeded with the correct number of molecules. In the first case, MOLREP found seven molecules, so that the final model has a spurious extra molecule. At present, the $M r B U M P$ pipeline does not deal explicitly with translational NCS, as occurs for example in OPPF651. In the majority of cases, there were several models and methods that could be used to solve the structure and the choice selected by the script (after identifying a 'success') or the author (after examining 'marginal' solutions) is largely arbitrary. Often, the structure could be solved both with the monomer search model and with a multimer. For example, BA0288 could be solved with chain $A$ of 1 ul1 or with the octamer downloaded from the PQS server. Both refine quickly to adequate $R$ values, though the constrained geometry of the octamer leads 
Table 6

Summary of results obtained by running AutoAMoRe on 18 target structures.

The column labels correspond to those in Table 5. If the $\mathrm{R} / \mathrm{Rf}$ columns are blank, the solution was rejected owing to excessive clashing. The information in italics was obtained after the workshop.

\begin{tabular}{|c|c|c|c|c|c|c|c|}
\hline$\underline{\text { Target }}$ & $N_{\text {mol }}$ & Model & $\begin{array}{l}\% \\
\text { ID } \\
\end{array}$ & $\begin{array}{l}\text { Initial } \\
\mathrm{R} / \mathrm{Rf}\end{array}$ & $\begin{array}{l}\text { Final } \\
\mathrm{R} / \mathrm{Rf}\end{array}$ & Success & Rebuilt \\
\hline BA0288 & 8 & $1 \mathrm{u} 11$ & 65 & $0.41 / 0.42$ & $0.32 / 0.35$ & $\mathrm{Y}$ & $\mathrm{Y}$ \\
\hline BA0296 & 2 & 1cli & 53 & $0.46 / 0.47$ & $0.35 / 0.48$ & & \\
\hline BA0592 & 6 & $1 \mathrm{pjc}$ & 55 & & & & \\
\hline BA1071 & 1 & 1ak1 & 73 & $0.42 / 0.41$ & $0.26 / 0.41$ & $\mathrm{Y}$ & $Y$ \\
\hline BA1483 & 6 & $1 e c p$ & 56 & $0.51 / 0.51$ & $0.39 / 0.46$ & Y & \\
\hline BA1563 & 2 & $1 v 8 f$ & 48 & $0.52 / 0.54$ & $0.43 / 0.52$ & $P$ & $Y$ \\
\hline BA3935_1 & 4 & $1 \mathrm{dhp}$ & 43 & $0.51 / 0.50$ & $0.41 / 0.46$ & $\mathrm{Y}$ & $Y$ \\
\hline BA3935_2 & 4 & $1 \mathrm{dhp}$ & 43 & $0.48 / 0.47$ & $0.36 / 0.43$ & $\mathrm{Y}$ & $Y$ \\
\hline BA4499 & 1 & $1 \mathrm{jr} 9$ & 70 & $0.43 / 0.49$ & $0.30 / 0.34$ & $\mathrm{Y}$ & $Y$ \\
\hline BA4508 & 1 & 1qum & 31 & $0.52 / 0.51$ & $0.39 / 0.52$ & & \\
\hline BA5696 & 2 & $1 j r 9$ & 55 & $0.44 / 0.44$ & $0.30 / 0.34$ & $Y$ & $Y$ \\
\hline BA5705 & 2 & $1 \mathrm{vrd}$ & 41 & & & & \\
\hline BSAppa & 1 & $1 \mathrm{dpp}$ & 26 & & & & \\
\hline BSYloQ & 1 & 1uol & 40 & $0.56 / 0.56$ & $0.39 / 0.60$ & & \\
\hline CJ0982 & 2 & $1 \mathrm{wdn}$ & 27 & & & & \\
\hline OPPF 651 & 2 & $1 p h p$ & 77 & $0.49 / 0.51$ & $0.34 / 0.48$ & $Y$ & \\
\hline OPPF1314 & 2 & $1 \mathrm{ydm}$ & 47 & $0.49 / 0.46$ & $0.37 / 0.46$ & $\mathrm{Y}$ & $\mathrm{Y}$ \\
\hline OPPF 2245 & 2 & $1 \mathrm{kon}$ & 36 & & & & \\
\hline
\end{tabular}

www.ccp4.ac.uk/martyn/BMP/mrbump. php and feedback is encouraged.

5.1.2. Automated molecular replacement with AutoAMoRe. The advantages of $A M o R e$ are its speed and flexibility. AutoAMoRe is a Python script produced as part of the $C C P 4$ automation project to automate the numerous steps in solving a structure by MR using AMoRe. The AutoAMoRe script calls various CCP4 utility programs. It checks the native Patterson for translational NCS and, if appropriate, positions molecules on a pairwise basis. The final coordinates are generated using PDBSET and checked for clashes with DISTANG. Auto$A M o R e$ generates a concise summary file of important parameters. The methodology adopted was to feed the target sequence into the $B L A S T$ server and choose as the template the solved structure with highest homology, after

to a slightly poorer result. The advantage of the multimeric search is speed and potentially signal-to-noise ratio and in general it would be tried first.

Of the 17 structures attempted (ignoring duplicate entries in Table 5), ten were essentially solved, six were possibly solved but require further investigation and one was clearly unsolved. It should be stressed that these conclusions are based on statistics from the MR and refinement programs and in some cases from rebuilding in $A R P / w A R P$ and are provisional pending structure completion. In the case of CJ0982, which is deemed unsolved, the initial homology search yields only one hit which has a sequence identity of $44 \%$ but with an alignment length of only 70 residues. As discussed elsewhere, there were problems with the data processing of OPPF1314 and the result quoted in Table 5 is against the original problematic data truncated to $2.3 \AA$ resolution.

The $M r B U M P$ package is still under development. The current version is expected to automate structure solution via MR in straightforward examples. For the current test cases, it identified solutions or likely solutions in the majority of cases. Many of these cases had good homologues available in the PDB and could be solved by any reasonable method. For these, the advantage of $M r B U M P$ is simply one of convenience, in particular when several homologues need to be tried and compared.

$M r B U M P$ requires the $C C P 4$ package plus a small number of helper applications and it was installed at York without problems. It is currently run from a simple shell script and users at the workshop found it easy to run the package for themselves. MrBUMP provides a framework within which further developments can be made in order to tackle more difficult cases. Ongoing work addresses both the algorithms applied in each step and the connecting work flow. Since the workshop, $\operatorname{MrBUMP}$ has been made available at http:// excluding any with $100 \%$ identity. The coordinates were downloaded from the EBI (http://www.ebi.ac.uk/) and passed through CHAINSAW. This selection and manipulation was performed manually and only the subsequent MR calculations with $A M o R e$ were automated. However, the AutoAMoRe script has since been incorporated as a module in the MrBUMP pipeline. AutoAMoRe was run on 18 of the target data sets using a monomer model in each case. Solutions were scored by inspecting the final correlation coefficient for all molecules in the asymmetric unit and any solution with greater than 20 clashes was rejected. The highest scoring solution was subjected to ten cycles of refinement using REFMAC with the default parameters from the $C C P 4 i$ GUI. If the $R_{\text {free }}$ fell by more than $5 \%$ during refinement, the solution was deemed to be successful. Six structures were solved successfully using the version of the AutoAMoRe software available at the workshop. A number of subsequent improvements to the software resulted in solutions to another four cases, an overall success rate of $55 \%$. The results are summarized in Table 6 .

5.1.3. BALBES. BALBES is a system for automatic $\mathrm{MR}$ developed by FL, AAV and GNM. It has three main components: the PDB (Bernstein et al., 1977; Berman et al., 2000), which has been reorganized to aid model selection, a Python script which controls the work flow and makes decisions and scientific programs to perform the actual calculations. The script uses the following programs: SFCHECK for structurefactor analysis, $M O L R E P$ for molecular replacement and REFMAC for refinement. Several other programs for purposes such as alignment and searching in the reorganized PDB have been developed.

The $\sim 30000$ structures in the PDB have been reorganized and classified according to sequence and three-dimensional structure. Redundant entries are removed if two proteins have a sequence identity above $90 \%$ or if the root-mean-square 
Table 7

Summary of results obtained using $B A L B E S$.

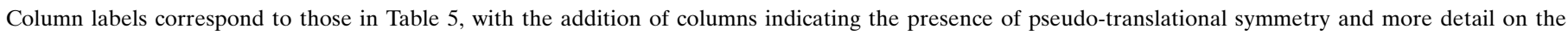
multimeric state and number of copies expected ( $N_{\text {mol }}$ expected) and found ( $N_{\text {mol }}$ found).

\begin{tabular}{|c|c|c|c|c|c|c|c|c|c|}
\hline Target & $N_{\text {res }}$ & Model & $\%$ ID & Pseudotranslation & $\begin{array}{l}N_{\text {mol }} \\
\text { expected }\end{array}$ & $\begin{array}{l}N_{\text {mol }} \\
\text { found }\end{array}$ & $\begin{array}{l}\text { Initial } \\
\mathrm{R} / \mathrm{Rf}\end{array}$ & $\begin{array}{l}\text { Final } \\
\mathrm{R} / \mathrm{Rf}\end{array}$ & Success $\dagger$ \\
\hline BA0288 & 161 & $1 \mathrm{xmp}, 1 \mathrm{o} 4 \mathrm{v}$ & 100 & $\mathrm{~N}$ & $4 \times 2$ & $4 \times 2$ & $0.31 / 0.31$ & $0.24 / 0.28$ & $\mathrm{Y}$ \\
\hline BA0592 & 377 & $1 \mathrm{pjc}$ & 54 & $\mathrm{~N}$ & 7 & 6 & $0.42 / 0.42$ & $0.30 / 0.36$ & $\mathrm{Y}$ \\
\hline BA1071 & 311 & $1 \mathrm{doz}$ & 72 & $\mathrm{~N}$ & 1 & 1 & $0.45 / 0.45$ & $0.29 / 0.39$ & $\mathrm{Y}$ \\
\hline BA1483 & 235 & $1 \mathrm{xe} 3$ & 100 & $\mathrm{~N}$ & $4 \times 2$ & $4 \times 2$ & $0.44 / 0.44$ & $0.22 / 0.27$ & $\mathrm{Y}$ \\
\hline BA1563 & 282 & $1 \mathrm{ufv}$ & 48 & $\mathrm{Y}$ & 2 & 2 & $0.58 / 0.56$ & $0.43 / 0.52$ & $\mathrm{Y} / \mathrm{M}$ \\
\hline BA3935_1 & 292 & $1 \mathrm{dhp}$ & 41 & $\mathrm{~N}$ & 4 & 4 & $0.52 / 0.52$ & $0.39 / 0.41$ & $\mathrm{Y}$ \\
\hline BA3935_2 & 292 & $1 \mathrm{dhp}$ & 41 & $\mathrm{~N}$ & 4 & 4 & $0.49 / 0.47$ & $0.33 / 0.38$ & $\mathrm{Y}$ \\
\hline BA4499 & 283 & $1 \mathrm{jr} 9$ & 70 & $\mathrm{~N}$ & 2 & 2 & $0.44 / 0.44$ & $0.29 / 0.34$ & $\mathrm{Y}$ \\
\hline BA4508 & 298 & 1qtw & 32 & $\mathrm{~N}$ & 2 & 1 & $0.52 / 0.54$ & $0.40 / 0.50$ & $\mathrm{Y} / \mathrm{M}$ \\
\hline BA5696 & 208 & $1 \mathrm{jr} 9$ & 55 & $\mathrm{~N}$ & 2 & 2 & $0.45 / 0.44$ & $0.29 / 0.33$ & $\mathrm{Y}$ \\
\hline BA5705 & 327 & 1 eep & 33 & $\mathrm{~N}$ & 2 & 2 & $0.55 / 0.56$ & $0.41 / 0.47$ & $\mathrm{Y}$ \\
\hline OPPF651 & 394 & $1 \mathrm{php}$ & 77 & $\mathrm{Y}$ & 2 & 2 & $0.65 / 0.65$ & $0.31 / 0.39$ & $\mathrm{Y}$ \\
\hline OPPF1294 & 193 & 1yby & 52 & $\mathrm{~N}$ & 2 & None & & & $\mathrm{N}$ \\
\hline OPPF1311 & 255 & $1 \operatorname{lm} 4$ & 39 & $\mathrm{Y}$ & $2 \times 2$ & 2 & $0.60 / 0.60$ & $0.56 / 0.63$ & $\mathrm{~N}$ \\
\hline OPPF1314 & 200 & $1 \mathrm{ydm}$ & 47 & $\mathrm{~N}$ & 2 & 2 & $0.49 / 0.48$ & $0.36 / 0.43$ & $\mathrm{Y}$ \\
\hline OPPF2088 & 139 & loqq & 26 & $\mathrm{~N}$ & 1 & 1 & $0.58 / 0.55$ & $0.49 / 0.59$ & $\mathrm{~N}$ \\
\hline OPPF2153 & 222 & $1 q u 0$ & 23 & $\mathrm{Y}$ & $2 \times 2$ & 2 & $0.69 / 0.70$ & $0.51 / 0.57$ & $\mathrm{P} / \mathrm{M}$ \\
\hline OPPF2245 & 229 & $1 \mathrm{kon}$ & 37 & $\mathrm{~N}$ & 2 & 2 & $0.54 / 0.55$ & $0.41 / 0.53$ & $\mathrm{P} / \mathrm{M}$ \\
\hline
\end{tabular}

$\dagger$ Y, definite solution; P, probable solution; N, no solution; M, checked manually.

deviation between matched atom pairs after superposition is less than $1 \AA$. This reduces the number of entries to a reference set of $\sim 10000$ structures, which are organized into a hierarchical database based on similarity. For each entry, potential multimers and domain structures are established and catalogued.

The Python script reads the experimental data and sequence information for the protein under investigation. The reorganized PDB is searched for related sequences, candidate models are identified and coordinates returned with multimers and domains where appropriate. The whole search takes around $10 \mathrm{~s}$ on a Macintosh G5 computer. The putative models are modified according to sequence identity and surface accessibility. The experimental data are analysed using $S F C H E C K$, which indicates problem features such as pseudotranslation, twinning or anisotropy and suggests the best resolution for the MR search. Information from these analyses is passed to MOLREP. Several protocols are tested in order: first with multimers, then with individual subunits and then with domains. After each protocol, a decision is made as to whether the 'solution' is correct. If the number of expected monomers is not yet satisfied, then MR is continued. During the workshop models were passed directly to $A R P / w A R P$ for rebuilding. Subsequently, a better protocol has been implemented: the MR solution is first passed to REFMAC for a number of cycles of rigid-body followed by restrained refinement. This has led to substantially better results for the rebuilding.

$B A L B E S$ is at the development stage and the database is updated automatically and regularly. For the current tests the database includes PDB entries released by the end of 2004.

Only a subset of the available protocols was necessary for the workshop examples. These included simple MR with one subunit (two cases, one successful), a search with dimers (four cases, two successful, one probably successful), a stepwise search for multiple subunits (13 cases, ten successful and one probable) and a use of pseudo-translation (four cases, two successful, one probable). More sophisticated protocols such as domain searches, multi-copy searches, iterative refinement and MR were not required for the current tests. Experience at the workshop suggested that implementation of several protocols needed to be faster. The results are summarized in Table 7.

\subsection{Summary of molecular-replacement pipelines}

Out of the structures considered, the majority have a close homologue available and are straightforward to solve by MR. The minority that are not straightforward to solve are the interesting examples for methods development and will be the focus of further work. The difficulty may arise from problems in the data processing. In other cases, more sophisticated model generation may be needed or experimental phasing is required.

The solution of the OPPF1314 structure was one of the key achievements of the workshop. All three pipelines were able to determine the solution with either the low-resolution data integrated using $D E N Z O / S C A L E P A C K$ or that processed using the three-dimensional XDS option of XIA-DPA. Refining and building a complete model proved more challenging and was only possible after reprocessing the data.

Comparison of the results with $M r B U M P$ and $B A L B E S$ at the workshop showed that even for identical models $A R P /$ $w A R P$ performed better with the $M r B U M P$ solutions. A key difference between the two approaches was the absence of an automated refinement step for $B A L B E S$ prior to attempting to rebuild the model with $A R P / w A R P$. The introduction of refinement into the $B A L B E S$ protocol subsequent to the 
Table 8

Rebuilding with REFMAC-ARP/wARP.

For each MR solution, both $A R P / w A R P$ and $p y W A R P$ were tried: $A R P / w A R P$ as an assessment of the quality of each MR solution and $p y W A R P$ for its own evaluation. Values correspond to the number of residues traced in the asymmetric unit, with the number of residues for which side chains are built in parentheses.

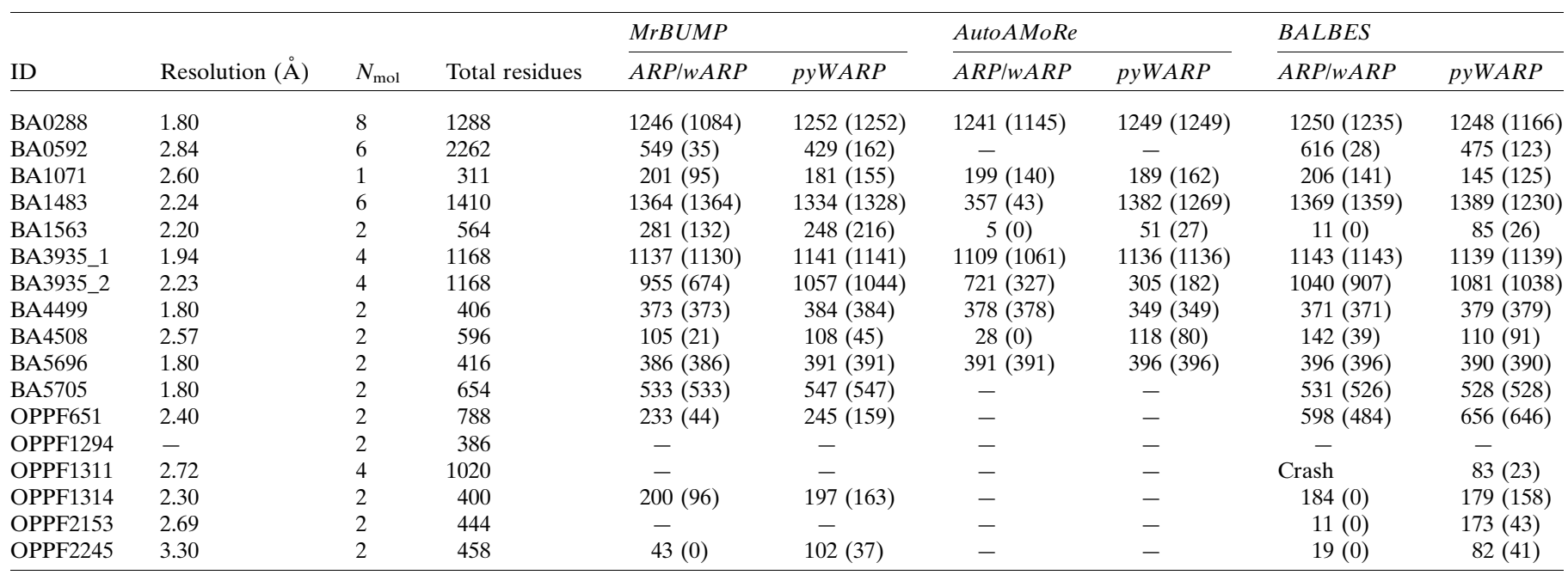

workshop now gives highly comparable results for the two pipelines (Tables 5 and 7).

At the simplest level, the role of automation is one of convenience, providing a solution with little user effort. However, it is likely that automation can provide more objective criteria of relative success for different models and also optimize the solution to minimize the effort required later for model rebuilding and completion.

\section{Experimental phasing}

Experimental phasing was not addressed in any depth as part of the automation testing, as the pipelines are at an earlier stage of development. Again, it became clear that many key items of information were not directly available, e.g. several reflection files did not provide correct wavelength information or document MAD data sets. OPPF1294, OPPF1311, OPPF2088 and OPPF2153 had all been solved previously using the SHELX suite. However, the SiaP structure was phased and largely built during the workshop, providing insight into how best to use weak phase information for automated model building at moderate resolution.

The principal software vehicle for this investigation was Pirate, a statistical phase-improvement program (Cowtan, 2000). Pirate classifies the electron density by sparseness/ denseness and order/disorder without requiring knowledge of the solvent content. Statistical targets are constructed, from which the distribution of probable density values is inferred on the basis of local density mean and variance. These targets are optimized to the problem at hand by the use of a known 'reference' structure which is manipulated by a process of scaling and error simulation to produce a map which is statistically similar to the map under examination. The software, which is still under development, is designed to be used in a fully automated manner.
The SiaP structure was phased using only the peak data from the Se sites found using SHELXD and initial phasing performed by SHELXE. This uses a solvent-flattening procedure to refine the initial SAD estimates and outputs phases and associated figures of merit. During the workshop, phases were recalculated using MLPHARE to record Hendrickson-Lattman (HL) coefficients. The average figure of merit was 0.43 , falling to 0.15 at $2.7 \AA$. Pirate was used to improve these SAD phases, reducing the overall phase error from 64 to $48^{\circ}$ (evaluated against the final model fully refined after the workshop) and providing better and much more realistic estimates of the figure of merit. Model completion was attempted from both these starting phase sets (§7.4).

\section{Constructing and completing models}

There was not sufficient time at the workshop to explore fully the best approach to the problem of model preparation. In cases where the resolution of the data set extends to $\sim 2.3 \AA$, packages such as $A R P / w A R P$ can often build a model automatically, providing that there are sufficiently good quality starting phases. $A R P / w A R P$ was run on all suitable MR solutions and the results are given in Table 8; this exercise was subsequently repeated using pyWARP (Cohen et al., 2004; Table 9). However, model building is a real stumbling block for lower resolution data sets and models with low sequence identity (typically less than $30 \%$ ). Full automation of the MR pipeline for low-resolution data may require the incorporation of new modules such as Buccaneer (Cowtan, 1998, 2001), which is designed to recognize larger structural features. In all cases it is necessary to complete the model using a graphical display and Coot (Emsley \& Cowtan, 2004) can provide this functionality. These modules are now described in more detail. 
Table 9

Rebuilding OPPF1314 with REFMAC-ARP/wARP.

After the OPPF1314 data were reprocessed, both ARP/wARP and $p y W A R P$ were applied to the two-dimensional (OPPF1314M) and three-dimensional (OPPF1314X) data sets at three different resolution cutoffs. The best results at $1.5 \AA$ are obtained from the three-dimensional integration, demonstrating better treatment of high mosaicity. For all tests, the extra functionality of pyWARP proved more successful. Column labels are equivalent to those in Table 8.

\begin{tabular}{|c|c|c|c|c|c|}
\hline \multirow[b]{2}{*}{ ID } & \multirow[b]{2}{*}{$\begin{array}{l}\text { Resolution } \\
\text { (§) }\end{array}$} & \multirow[b]{2}{*}{$N_{\text {mol }}$} & \multirow[b]{2}{*}{$\begin{array}{l}\text { Total } \\
\text { residues }\end{array}$} & \multicolumn{2}{|l|}{$B A L B E S$} \\
\hline & & & & $A R P / w A R P$ & $p y W A R P$ \\
\hline OPPF1314X & 1.5 & 2 & 400 & 193 (109) & $294(279)$ \\
\hline OPPF1314M & 1.5 & 2 & 400 & $131(34)$ & $257(246)$ \\
\hline OPPF1314X & 1.65 & 2 & 400 & 277 (268) & $322(316)$ \\
\hline OPPF1314M & 1.65 & 2 & 400 & 253 (208) & 303 (303) \\
\hline OPPF1314X & 1.85 & 2 & 400 & 271 (249) & $323(323)$ \\
\hline OPPF1314M & 1.85 & 2 & 400 & $261(226)$ & $322(322)$ \\
\hline
\end{tabular}

\section{1. $A R P / W A R P$}

$A R P / w A R P$ was used to evaluate the quality of the solutions obtained from the different MR pipelines. Each solution was input to the currently distributed version of $A R P / w A R P$ (v.6.1.1) and ten rebuilding cycles were performed (each comprising five update cycles) starting from the positioned model (using the mode described in Perrakis et al., 1999, 2001). In this procedure, most of the stereochemical information is preserved as long as possible during the building, giving the refinement program $R E F M A C$ more restraints.

Some of the data sets highlighted an error in the sequencedocking/side-chain building module of $A R P / w A R P$, which occurred when a main-chain fragment became longer than the provided sequences. This was fixed following the meeting and the corrected version used to generate Tables 8 and 9. All solutions now run through to the preset end, with the exception of the $B A L B E S$ solution for OPPF1311, which still causes problems.

The available MR solutions were used to evaluate pyWARP, a new control system for $A R P / w A R P$ currently under development (Cohen et al., 2004). This control system makes run-time decisions based on the current status of the model. Tables 8 and 9 also show the results from pyWARP, which appears to perform a little better than $A R P / w A R P$ and clearly docks a greater portion of the traced main chain into the sequence. Indeed, in difficult cases (OPPF2153 and OPPF2245) pyWARP significantly improved the completeness of the autotraced model, showing the value of using variable parameterization during the procedure.

\subsection{Buccaneer}

Buccaneer is a new model-building program which makes repeated application of a single optimized feature-recognition technique. The process involves the construction of an optimal likelihood density target for the electron density in a $4 \AA$ sphere around a typical $\mathrm{C}^{\alpha}$ atom (the idea, but not the target function, is similar to that in Ioerger \& Sacchettini, 2002). The 'optimization' of the target is similar to that described in $\$ 6$. A six-dimensional search is made in the unsolved map for likely $\mathrm{C}^{\alpha}$ atom positions using Fast Fourier Feature Recognition (Cowtan, 2001). Once initial candidate positions are obtained, a 'growing' procedure is applied to find chain fragments. New residues are added in each direction using the Ramachandran plot to constrain chain geometry and a two-residue-deep search to rank the fit to density of the new positions. It is computed using the same likelihood density target, but now calculated in real space. The procedure continues until the fit to density falls below some threshold. The next stage is to combine and merge overlapping chain fragments, using the Coot utility GLOBULARISE-PROTEIN. The approach is implemented using the CLIPPER libraries (Cowtan, 2003). Despite its simplicity, it quickly rebuilt missing features for two of the lower resolution MR structures, BA1071 and BA4508. Its performance with the SiaP structure is described below. It can be integrated into a recycling scheme including density modification and refinement. Other improvements are possible, such as using bi-residue groups in common conformations.

\subsection{Coot}

Coot is a molecular-graphics application for protein map interpretation and structure validation. The workshop highlighted a number of its strengths, but also some missing features. It proved very powerful for rebuilding initial models from automated model building. The validation tools identify poorly built regions of the model, both by a variety of geometrical indicators and by fit-to-density analysis. These regions can be rapidly improved by interactive real-space refinement and regularization. Although Coot was not used greatly in its role as a model-completion and validation tool by this workshop, missing features were highlighted including a means to reverse a baton-built $\mathrm{C}^{\alpha}$ trace, better tools for joining fragments, a user interface for the automatic restoration of side chains and tools to correct out-of-register errors (a more substantial problem). Some of these deficiencies have now been addressed. Coot presently implements all these algorithms from a graphical interface, but it should be possible to incorporate the underlying functionality into a command-linedriven program suitable for an automated pipeline.

\subsection{Experimental phasing case history: SiaP}

Several attempts were made to rebuild this structure using $A R P / w A R P$. In $\$ 2.2$ we describe the results obtained before the workshop. The breakthrough came from the procedures described in $\$ 6$, namely when the SAD experimental phase distributions in the form of the Hendrickson-Lattman coefficients calculated using the MLPHARE program were provided as restraints to REFMAC-ARP/wARP. Firstly, the procedure was started from the RESOLVE partial model and the MLPHARE phases and within 25 cycles it had built 560 of the expected 612 residues, with 545 side chains docked. Secondly, the more straightforward approach of feeding the Hendrickson-Lattman coefficients directly into the $A R P /$ $w A R P$ procedure used for building the initial model was 
attempted. This took longer, but effectively reached the same solution. The third and fourth tests used Buccaneer to construct an initial model. The third test began from the MLPHARE phases, from which Buccaneer built a polyalanine model of 288 residues ( $47 \%$ of the total). The fourth test used the improved phases from Pirate and with these Buccaneer was able to construct a 384-residue polyalanine model (63\%). Both these models were able to kick-start the ARP/wARP procedure and speeded up its convergence considerably. Starting from the Pirate/Buccaneer model, ARP/wARP completely built 578 residues (94\%).

With the current state of developments this is an impressive result: the automated building and refinement of an essentially complete protein structure with rather weak $2.6 \AA \mathrm{SAD}$ data. The application of any specific program in this solution is certainly less important than the retention of the full experimental phase distribution as restraints in the model-building/ refinement stage.

This result has influenced several developments within the CCP4 and York automation pipelines currently under construction.

(i) Experimental phase restraints, in the form of Hendrickson-Lattman coefficients, were essential to keep the $A R P / w A R P$-REFMAC cycles on target.

(ii) Phase improvement using Pirate improved this. It is important that the weighting of the experimental phases is realistic.

(iii) While initial model building using feature recognition such as Buccaneer or RESOLVE was not able to generate a complete structure, starting models were created which considerably speeded up the ARP/wARP-REFMAC process.

\subsection{Molecular-replacement case history: OPPF1314}

Before the workshop, the DENZO/SCALEPACK data set from the high-resolution data-collection pass alone had given a clear MR solution with the expected two molecules in the asymmetric unit. Structure completion with $A R P / w A R P$ was partly successful, but convergence was slow: missing data at low-resolution inevitably degrade the electron density which $A R P / w A R P$ requires for selection and rejection of atomic sites.

After initial reprocessing with the $X I A-D P A$ three-dimensional option, the data from the combined high- and lowresolution passes showed an expected distribution in reflection intensities (\$4.3; Fig. 1). This allowed $A R P / w A R P$ to produce a structure containing 329 residues in nine chains out of the 400 residues expected in the asymmetric unit (for the final cycle of rebuilding the $R$ factor was 0.234 with an $R_{\text {free }}$ of 0.289 ). The maps showed that one part of each molecule was poorly ordered, explaining the missing residues. Further inspection of the maps revealed additional features in the electron density that were not part of the protein and could be attributed to bound ligands (\$7.6).

The two-dimensional integrated data also led to a similarly successful result (Table 9) after reprocessing using the improved XIA-DPA software (\$4.3). However, with data from both the three-dimensional and two-dimensional integration $A R P / w A R P$ built more residues when the data were restricted to $1.85 \AA$ resolution rather than using the full range to $1.5 \AA$. This may reflect the optimization of $A R P / w A R P$, the poorer quality of the outer shells or be the result of residual errors arising from the effect of the mosaicity on the highest resolution data. In all cases, the extra functionality of $p y W A R P$ proved its worth.

Since the workshop, refinement of this structure has been completed, giving a model containing 192 residues from each chain, one ADP bound to each chain and 256 modelled waters. The current $R$ factor is 0.219 (with an $R_{\text {free }}$ of 0.265 ).

What conclusions can be drawn from this case study?

(i) The importance of data quality and flagging of unexpected values of the quality-assessment parameters at the data-processing stage (see $\S 4.3 .1$ ).

(ii) The importance of completeness of low-resolution data for electron density.

(iii) The robustness of MR methods even with substandard data.

(iv) The value of CHAINSAW-type procedures before the MR search and after MR solution but before rebuilding.

(v) The automated updating of parameters during the course of model rebuilding using pyWARP led to significantly better performance compared with the normal ARP/wARP procedure.

(vi) A good test for ligand fitting (§7.6).

\subsection{Ligand binding to OPPF1314}

7.6.1. ARP/WARP LigandBuild. Once a model is close to completion, the remaining density can be searched for smallmolecule ligands. This procedure can be accomplished using the $A R P / w A R P$ suite (v.6.1.1) with $C C P 4$ and a text editor. The ARP/wARP LigandBuild graphical user interface requires structure-factor amplitudes, protein coordinates without any HETATM entries (used to generate a mask) and a set of coordinates for the known ligand (Zwart et al., 2004).

For OPPF1314, the extra density after model building (\$7.5) was assumed to be attributable to one or both of the cofactors in the crystallization screen, ATP and 5-formyltetrahydrofolate, which have a somewhat similar shape. Automated ligand fitting was attempted for both cofactors. The first and second trials failed; the map was still too noisy and wrong sites with impossible conformations were found. In each case, the volume covered by these was then added to the mask and the procedure was repeated. The correct sites were found in the third and fourth attempts and were verified by inspection of the electron density. The fit with ATP was clearly superior and after inspection of the electron density it was concluded that each of the two molecules in the asymmetric unit bound a well ordered ADP (Fig. 2). Although there were other residual density features, they could not be unambiguously attributed to 5-formyltetrahydrofolate.

The result showed the need for updating masks. The PDB file was modified automatically to add extra 'atoms' at each 
cycle. This is also needed when searching for multiple ligands, where it is recommended to build the largest first. It also showed how the recognition capabilities of the software are limited by noise in the map, as the search currently only checks a limited number of features for a potential ligand site.

7.6.2. Ligand building with Coot. Coot has the ability to search a map for likely ligand sites. It uses the REFMAC monomer dictionary to provide a description of the ligand geometry and also needs a set of coordinates for the known ligand, which can be provided by the $C C P 4$ program LIBCHECK (Vagin et al., 1998). As with ARP/wARP LigandBuild, the density is masked by selected coordinate sets. After ARP/wARP for OPPF1314, Coot found seven putative ligand sites matching the expected size and shape for ADP. On visual inspection, several were found to be protein structure missing from the model, but the first and second sites ordered on the density correlation corresponded to the two nucleotide sites. The fit to density was then optimized using Coot's real-space refinement option.

\subsection{Summary of model rebuilding}

From Tables 8 and 9, it is noticeable that the success of $A R P / w A R P$ varies substantially depending on how the positioned model was obtained. For target BA1563, for example, $A R P / w A R P$ was able to rebuild about half of the model using the output of $M r B U M P$, while very little could be rebuilt from the other putative solutions. Both $M r B U M P$ and BALBES used 1 ufv as the template and MOLREP for MR. At the workshop, for target BA3935_2 ARP/wARP rebuilt about $80 \%$ of the model from $M r B U M P$, but only about $50 \%$ of the model from $B A L B E S$. Post mortem analysis showed that the

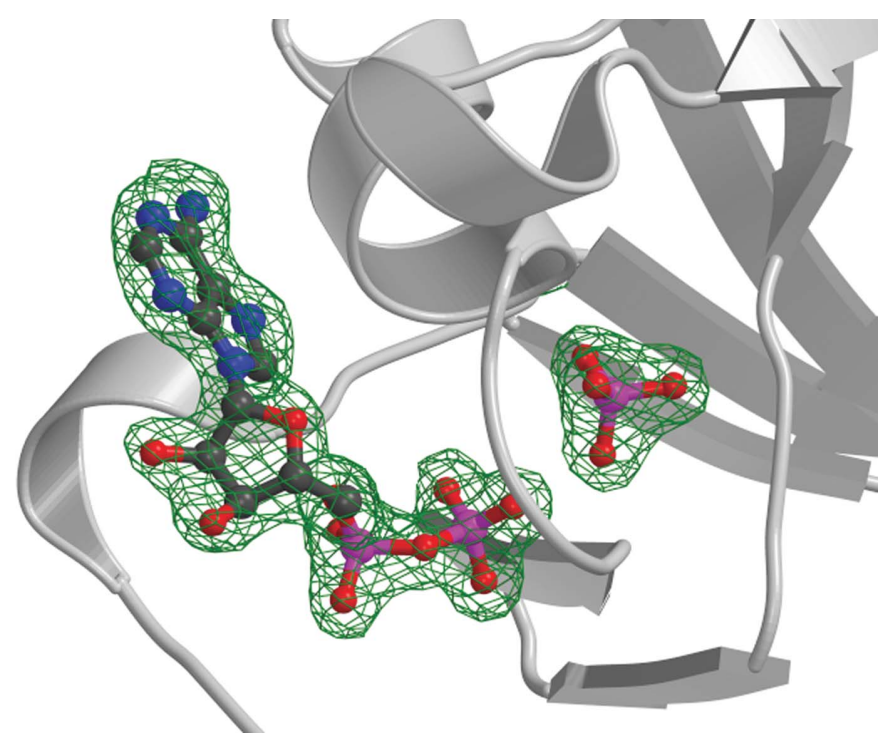

Figure 2

Part of the model for OPPF1314 showing the bound cofactor ADP (with a separate phosphate group presumably resulting from ATP hydrolysis) after automatic fitting of the cofactor by either $A R P / w A R P$ or Coot and further refinement. Green contours show the OMIT-map density for the cofactor contoured at $3 \sigma$. This figure was drawn using BobScript (Esnouf, 1999) and rendered with Raster3D (Merritt \& Murphy, 1994). difference was that $\operatorname{MrBUMP}$ carried out 30 cycles of $R E F M A C$ refinement before $A R P / w A R P$. This refinement step has subsequently been activated in $B A L B E S$.

We have not carried out a systematic investigation of the factors which are important for successful rebuilding and the differences noted may be coincidental. However, where there is no clear-cut MR result, there is a clear advantage in taking several putative solutions through to model rebuilding. In the BA3935_2 example, both templates 1dhp and 1s5t have $42 \%$ sequence identity with the target and both should be tried. The high-resolution limit of the data for BA1563 and BA3935_2 is in both cases $2.2 \AA$ and it is in this regime where subtle differences may affect the $A R P / w A R P$ procedure. Automated schemes are particularly useful for investigations of such multiple models.

\section{Conclusions}

Protein crystallography has a series of potential bottlenecks, including protein overexpression, solubility, crystallization and structure solution. Recently, rapid advances in the first three of these have been made (see other contributions to this issue). Considerable progress has been and is being made worldwide in the automation of structure analysis. The automation of image processing and data reduction was addressed at the workshop using XIA-DPA. The results obtained emphasized the importance of this step and showed that while it was in principle subject to a high level of automation, great care needs to be taken in establishing protocols and in passing the appropriate information to subsequent steps.

The MR step in the structure-solution pipeline is presently closest to full automation. Three emerging procedures were extensively tested with a high success rate and this software should be released for general use within the next year. Lessons learnt at the workshop included (i) the apparent advantage of running several cycles of restrained refinement on correctly positioned MR models before starting the rebuilding procedure and (ii) generally trying a multimer as a model when appropriate before trying individual subunits. Based on the results obtained, automated MR procedures are likely to be successful, at least for crystals which diffract to $2.5 \AA$ or better and satisfy a number of defined criteria (overall $R_{\text {merge }} \simeq 6 \%$, low-resolution shell $\simeq 4 \%$, high-resolution shell $\simeq 35 \%$, completeness $\simeq 90 \%$ ). At present, twinning poses real problems, but this should be resolved in the near future: for crystals with merohedral twinning, diffraction to $\sim 2.1 \AA$ or better may be necessary.

Models for MR should satisfy one of the following criteria.

(i) $\sim 30 \%$ identity for one molecule in the asymmetric unit and no significant domain movement.

(ii) $\sim 45 \%$ identity for multiple molecules in the asymmetric unit and no significant domain movement.

(iii) $>50 \%$ identity for two or more molecules in the asymmetric unit, where there is significant domain movement.

For structures solved by experimental phasing, there are already modules such as the SHELX suite, AUTOSHARP and SOLVE/RESOLVE which integrate parts of the pipelines. 
These were tested on a couple of examples at the workshop and the importance of making full use of the HendricksonLattman coefficients for low-resolution data became clear. Pirate was tested for density modification and appears to provide a more realistic set of Hendrickson-Lattman coefficients than earlier software. Pipelines being developed are still at the early stages, but considerable insight was gained as to the direction which these developments should take. The restrictions on data quality are quite different from those stated above for MR; experimental phasing is effective at substantially lower resolutions but requires much more accurate estimates of intensity, usually achieved by measuring high-multiplicity data sets.

$A R P / w A R P$ was the only automated model-building tool used extensively. It proved to be very powerful for structures with data extending to $2.3 \AA$ or better (see Table 8). At lower resolutions problems were encountered and the featurerecognition tools within the Buccaneer and Coot programs, briefly tested during the workshop, would need to be exploited for these problems. However, crystals with diffraction limits in the greyzone (2.7-3.3 $\AA$ ) still require a lot of time and effort and sometimes this effort fails. High-throughput structure determination means a limited amount of time can be dedicated to an individual project, resulting in a need for automation. We have encountered several examples, BA0592 (from the workshop set) and BA4525 (collected recently), where crystals have been obtained, data collected and a MR solution found, but the project has had to be abandoned because automated model building and refinement failed. The more sustained effort of project-oriented research may have led to success.

Taken altogether, the outlook is very promising for modules for fully automated solution of protein crystal structures in the near future, provided the data are of sufficient quality and resolution.

The SPINE project is funded by the European Commission as SPINE (Structural Proteomics In Europe) contract No. QLG2-CT-2002-00988 under the Integrated Programme 'Quality of Life and Management of Living Resources'. GW and RK are supported by the BBSRC e-HTPX grant (BEP17782) and CB, NS, MGWT and MW by CCP4. KDC is supported by The Royal Society (grant No. 003R05674). PE and AAV are funded by BBSRC grant No. 87/B17320. GNM is supported by the Wellcome Trust, FL by the EU BIOXHIT contract under the Sixth Framework Programme thematic area 'Life Sciences, Genomics and Biotechnology for Health' contract No. LHSG-CT-2003-503420. ARP/wARP algorithm development at the NKI (AP, SXC) and the EMBL (VL, GL) is funded by the NIH (grant R01 GM62612-01) and the EU BIOXHIT contract. AP and SXC thank Marouane Ben Jelloul for his work in the development of $p y W A R P$.

\section{References}

Alzari, P. M. et al. (2006). Acta Cryst. D62, 1103-1113.

Bernstein, F. C., Koetzle, T. F., Williams, G. J. B., Meyer, E. F. Jr, Brice, M. D., Rodgers, J. R., Kennard, O., Shimanouchi, T. \& Tasumi, M. (1977). J. Mol. Biol. 112, 535-542.

Berman, H. M., Westbrook, J., Feng, Z., Gilliland, G., Bhat, T. N., Weissig, H., Shindyalov, I. N. \& Bourne, P. E. (2000). Nucleic Acids Res. 28, 235-242.

Brenner, S. E., Chothia, C. \& Hubbard, T. J. P. (1998). Proc. Natl Acad. Sci. USA, 95, 6073-6078.

Cohen, S. X., Morris, R. J., Fernandez, F. J., Ben Jelloul, M., Kakaris, M., Parthasarathy, V., Lamzin, V. S., Kleywegt, G. J. \& Perrakis, A. (2004). Acta Cryst. D60, 2222-2229.

Collaborative Computational Project, Number 4 (1994). Acta Cryst. D50, 760-763.

Cowtan, K. (1998). Acta Cryst. D54, 750-756.

Cowtan, K. (2000). Acta Cryst. D56, 1612-1621.

Cowtan, K. (2001). Acta Cryst. D57, 1435-1444.

Cowtan, K. (2003). IUCr Comput. Commun. Newsl. 2, 4-9.

Emsley, P. \& Cowtan, K. (2004). Acta Cryst. D60, 2126-2132.

Esnouf, R. M. (1999). Acta Cryst. D55, 938-940.

Evans, P. (1993). Proceedings of the CCP4 Study Weekend. Data Collection and Processing, edited by L. Sawyer, N. Isaacs \& S. Bailey, pp. 114-122. Warrington: Daresbury Laboratory.

Evans, P. (2006). Acta Cryst. D62, 72-82.

French, S. \& Wilson, K. (1978). Acta Cryst. A34, 517-525.

Huennekens, F. M., Henderson, G. B., Vitols, K. S. S. \& Grimsha, C. E. (1984). Adv. Enzyme Regul. 22, 3-13.

Ioerger, T. R. \& Sacchettini, J. C. (2002). Acta Cryst. D58, $2043-$ 2054.

Kabsch, W. (1993). J. Appl. Cryst. 26, 795-800.

Leslie, A. (1999). Acta Cryst. D55, 1696-1702.

McCoy, A. J., Grosse-Kunstleve, R. W., Storoni, L. C. \& Read, R. J. (2005). Acta Cryst. D61, 458-464.

Meier, C., Carter, L. G., Esnouf, R. M., Owens, R. J. \& Stuart, D. I. (2006). In preparation.

Merritt, E. A. \& Murphy, M. E. P. (1994). Acta Cryst. D50, 869873.

Murshudov, G. N., Vagin, A. A. \& Dodson, E. J. (1997). Acta Cryst. D53, 240-255.

Murzin, A. G., Brenner, S. E., Hubbard, T. \& Chothia, C. (1995). J. Mol. Biol. 247, 536-540.

Navaza, J. (1994). Acta Cryst. A50, 157-163.

Otwinowski, Z. \& Minor, W. (1997). Methods Enzymol. 276, 307-326.

Perrakis, A., Harkiolaki, M., Wilson, K. S. \& Lamzin, S. (2001). Acta Cryst. D57, 1445-1450.

Perrakis, A., Morris, R. \& Lamzin, V. S. (1999). Nature Struct. Biol. 6, 458-463.

Sauter, N., Grosse-Kunstleve, R. \& Adams, P. (2004). J. Appl. Cryst. 37, 399-409.

Schneider, T. R. \& Sheldrick, G. M. (2002). Acta Cryst. D58, 17721779.

Schwarzenbacher, R., Godzik, A., Grzechnik, S. K. \& Jaroszewski, L. (2004). Acta Cryst. D60, 1229-1236.

Terwilliger, T. C. (2003). Methods Enzymol. 374, 22-37.

Vagin, A. A., Murshudov, G. N. \& Strokopytov, B. V. (1998). J. Appl. Cryst. 31, 98-102.

Vagin, A. A. \& Teplyakov, A. (1997). J. Appl. Cryst. 30, 1022-1025.

Vaguine, A. A., Richelle, J. \& Wodak, S. (1999). Acta Cryst. D55, 191-205.

Zwart, P. H., Langer, G. G. \& Lamzin, V. S. (2004). Acta Cryst. D60, 2230-2239. 